\title{
Headache complaints increase the risk for temporomandibular disorder among Brazilian adolescents: A population-based study
}

\author{
Ana L. Franco, DDS. MSc'; Giovana Fernandes, DDS. MSc'; Daniela Gonçalves, AG. DDS. MSc. PhD²; \\ Cinara Camparis, M. DDS. MSc. PhD²; Richard Ohrbach, DDS. PhD. ${ }^{3}$ \\ 'Post Graduate student; ${ }^{2}$ Professor - Discipline of Temporomandibular Disorders and Orofacial Pain, \\ Department of Dental Materials and Prosthodontics, Faculdade de Odontologia de Araraquara, Unesp - Univ \\ Estadual Paulista. ${ }^{3}$ Associate Professor - Department of Oral Diagnostic Sciences, University at Buffalo, NY \\ Franco AL, Fernandes G, Gonçalves D, Camparis C, Ohrbach R. Headache complaints increase the risk for \\ temporomandibular disorder among brazilian adolescents: A population-based study. \\ Headache Medicine. 2012;3(4):217-20
}

\section{INTRODUCTION}

Headaches $(\mathrm{HA})$ are one of the most frequent symptoms observed in children and adolescents diagnosed with temporomandibular disorders (TMD).(1) A Brazilian research with adolescents from a tertiary care center found a significant increase in signs and symptoms of TMD in those with $\mathrm{HA}^{(2)}$ similarly to what was recently observed in adults. ${ }^{(3)}$

Although the association between HA and TMD has been studied in adolescents, results are not yet conclusive, in part because of methodological design. Indeed, there is a lack of studies in population-based samples, and in most cases, TMD pain characteristics are not explored deeply.

\footnotetext{
AIM

The aim of the present study was to verify if headache complaints (HAc) are associated to TMD diagnosis and pain characteristics, by using standardized methods of sampling and data collection in a population-based sample of adolescents.
} 


\section{MATERIALS AND METHODS}

The sample consisted of public school students, 12 to 14 years age, from Araraquara - SP, Brazil. Overall, 24 public schools were visited and 3,117 adolescents were invited to participate of the present study.

This study received full approval of the Research Ethic Committee of Faculdade de Odontologia de Araraquara, UNESP - Universidade Estadual Paulista (process \# 70/10).

The Research Diagnostic Criteria for Temporomandibular Disorders (RDC/TMD) ${ }^{(4)}$ Axis I (intraexaminer kappa values 0.529-0.884) and questions \#3 (Have you had pain in the face, jaw, temple, in front of the ear or in your ear in the past month?), \#4 (How many time did your facial pain begin for the first time?), \# 14 (Have you ever had your jaw lock or catch so that it won't open all the way) and \# 18 (During the last six months have you had a problem with headaches or migraines? - kappa $=0.688$ ) of Axis II questionnaire were used to collect data.

\section{RESULTS}

The sample consisted of 1,307 individuals, $56.8 \%$ $(n=742)$ girls. Among them, $80.7 \%(n=599)$ already presented menarche, mean age was 12.72 years. Overall, $397(30.4 \%)$ adolescents were diagnosed with TMD and 595 (45.5\%) presented HAc.

Comparing to boys, girls showed higher risks for presenting both, TMD and HAc (Relative Risk (RR) = 1.39; 95\% Confidence Interval $(\mathrm{Cl})=1.15-1.67)$, significantly those with menarche (1.38; 1.16-1.66) (Table 1).

Individuals reporting $\mathrm{HAc}$ presented higher risks for TMD (1.88; 1.62-2.17). The risk was particularly significant for painful TMD diagnosis $(1.51 ; 1.40-1.62)$ (Table 2).

Regarding TMD painful subtypes, risks were higher for combined (muscle and joint) painful TMD $(1.24 ; 1.18$ 1.31), followed for muscle TMD (1.23; 1.16-1.30) and joint TMD (1.04; 1.01-1.07) (Table 3).

Considering chronicity, not only HAc slightly increased the risk for acute TMD pain (1.09; 1.05-1.14), but also

\begin{tabular}{|c|c|c|c|c|c|c|c|c|}
\hline \multicolumn{9}{|c|}{ Gender / menarche } \\
\hline $\begin{array}{c}\text { TMD/ HAc } \\
\text { groups }\end{array}$ & $\begin{array}{l}\text { Boys } \\
\mathrm{n}(\%)\end{array}$ & $\begin{array}{c}\text { Girls Overall } \\
\mathrm{n}(\%)\end{array}$ & $\begin{array}{c}\mathrm{RR} \\
(95 \% \mathrm{Cl})\end{array}$ & $\begin{array}{c}\text { Girls (-) } \\
\text { menarche } \mathrm{n}(\%)\end{array}$ & $\begin{array}{c}\mathrm{RR} \\
(95 \% \mathrm{Cl})\end{array}$ & $\begin{array}{c}\text { Girls }(-) \\
\text { menarche } \mathrm{n}(\%)\end{array}$ & $\begin{array}{c}\mathrm{RR} \\
(95 \% \mathrm{Cl})\end{array}$ & $\begin{array}{l}\text { Total } \\
\mathrm{n}(\%)\end{array}$ \\
\hline $\begin{array}{l}-T M D \\
-H A C\end{array}$ & $\begin{array}{c}282 \\
(49.9)\end{array}$ & $\begin{array}{l}296 \\
(50.1)\end{array}$ & (ref.) & $\begin{array}{c}55 \\
(38.5)\end{array}$ & (ref.) & $\begin{array}{c}241 \\
(40.2)\end{array}$ & (ref.) & $\begin{array}{c}578 \\
(40.2)\end{array}$ \\
\hline $\begin{array}{l}+ \text { TMD } \\
-\mathrm{HAC}\end{array}$ & $\begin{array}{c}62 \\
(11.0)\end{array}$ & $\begin{array}{c}72 \\
(89.0)\end{array}$ & $\begin{array}{c}1.054 \\
(0.8627-1.289) \\
p=0.6320\end{array}$ & $\begin{array}{c}15 \\
(10.5)\end{array}$ & $\begin{array}{c}1.039 \\
(0.9221-1.171) \\
p=0.5027\end{array}$ & $\begin{array}{c}57 \\
(9.5)\end{array}$ & $\begin{array}{c}1.035 \\
(0.8561-1.251) \\
p=0.7603\end{array}$ & $\begin{array}{c}134 \\
(10.3)\end{array}$ \\
\hline $\begin{array}{l}-T M D \\
+H A c\end{array}$ & $\begin{array}{c}129 \\
(22.8)\end{array}$ & $\begin{array}{c}203 \\
(77.2)\end{array}$ & $\begin{array}{c}1.256 \\
(1.071-1.472) \\
{ }^{*} p=0.0045\end{array}$ & $\begin{array}{c}47 \\
(32.9)\end{array}$ & $\begin{array}{c}1.142 \\
(1.032-1.263) \\
{ }^{*} p=0.0072\end{array}$ & $\begin{array}{c}156 \\
(26.0)\end{array}$ & $\begin{array}{c}1.191 \\
(1.025-1.384) \\
{ }^{*} p=0.0224\end{array}$ & $\begin{array}{c}332 \\
(25.4)\end{array}$ \\
\hline $\begin{array}{l}+ \text { TMD } \\
+\mathrm{HAc}\end{array}$ & $\begin{array}{c}92 \\
(16.3)\end{array}$ & $\begin{array}{c}171 \\
(83.7)\end{array}$ & $\begin{array}{c}1.395 \\
(1.159-1.678) \\
{ }^{*} p=0.0002\end{array}$ & $\begin{array}{c}26 \\
(18.2)\end{array}$ & $\begin{array}{c}1.0793 \\
(0.9645-1.194) \\
p=0.1647\end{array}$ & $\begin{array}{c}145 \\
(24.2)\end{array}$ & $\begin{array}{c}1.389 \\
(1.162-1.660) \\
{ }^{*} p=0.0001\end{array}$ & $\begin{array}{c}263 \\
(20.1)\end{array}$ \\
\hline Total & $\begin{array}{c}565 \\
(100.0)\end{array}$ & $\begin{array}{c}742 \\
(100.0)\end{array}$ & & $\begin{array}{c}143 \\
(100.0)\end{array}$ & & $\begin{array}{c}599 \\
(100.0)\end{array}$ & & $\begin{array}{c}1307 \\
(100.0) \\
\end{array}$ \\
\hline
\end{tabular}

ref.= reference values. ${ }^{*}$ chi-square test

Table 2. Risk for painful TMD according to HAC

\begin{tabular}{|c|c|c|c|c|c|c|c|c|}
\hline \multicolumn{9}{|c|}{ Painful TMD } \\
\hline $\mathrm{HAC}$ & $\begin{array}{l}\text { No TMD } \\
\mathrm{n}(\%)\end{array}$ & $\begin{array}{c}\text { TMDOverall } \\
\mathrm{n}(\%)\end{array}$ & $\begin{array}{c}\mathrm{RR} \\
(95 \% \mathrm{Cl})\end{array}$ & $\begin{array}{c}\text { Non painful TMD } \\
n(\%)\end{array}$ & $\begin{array}{c}\mathrm{RR} \\
(95 \% \mathrm{Cl})\end{array}$ & $\begin{array}{c}\text { Painful TMD } \\
\mathrm{n}(\%)\end{array}$ & $\begin{array}{c}\mathrm{RR} \\
(95 \% \mathrm{Cl})\end{array}$ & $\begin{array}{l}\text { Total } \\
\text { n (\%) }\end{array}$ \\
\hline no & $\begin{array}{l}578 \\
(63.5)\end{array}$ & $\begin{array}{c}134 \\
(33.8)\end{array}$ & (ref.) & $\begin{array}{c}48 \\
(71.6)\end{array}$ & (ref.) & $\begin{array}{c}86 \\
(26.1)\end{array}$ & (ref.) & $\begin{array}{c}712 \\
(54.5)\end{array}$ \\
\hline yes & $\begin{array}{c}332 \\
(36.5)\end{array}$ & $\begin{array}{c}263 \\
(66.2)\end{array}$ & $\begin{array}{c}1.882 \\
(1.626-2.178) \\
{ }^{*} p<0.0001\end{array}$ & $\begin{array}{c}19 \\
(28.4)\end{array}$ & $\begin{array}{c}0.9762 \\
(0.9438-1.010) \\
p=0.1901\end{array}$ & $\begin{array}{c}244 \\
(73.9)\end{array}$ & $\begin{array}{c}1.510 \\
(1.400-1.629) \\
{ }^{*} p<0.0001\end{array}$ & $\begin{array}{c}595 \\
(45.5)\end{array}$ \\
\hline
\end{tabular}

ref.= reference values. *chi-square test 


\begin{tabular}{|c|c|c|c|c|c|c|c|c|c|c|}
\hline \multicolumn{11}{|c|}{ Painful TMD subtypes } \\
\hline $\mathrm{HAC}$ & $\begin{array}{c}\text { No TMD } \\
\text { +non painful } \\
\text { TMD } \\
\mathrm{n}(\%)\end{array}$ & $\begin{array}{c}\text { Painful TMD } \\
\text { overall } \\
\text { n (\%) }\end{array}$ & $\begin{array}{c}\mathrm{RR} \\
(95 \% \mathrm{Cl})\end{array}$ & $\begin{array}{c}\text { Painful } \\
\text { TMD } \\
\text { joint n (\%) }\end{array}$ & $\begin{array}{c}\mathrm{RR} \\
(95 \% \mathrm{Cl})\end{array}$ & $\begin{array}{c}\text { Painful } \\
\text { TMD } \\
\text { muscle } \\
n(\%)\end{array}$ & $\begin{array}{c}\mathrm{RR} \\
(95 \% \mathrm{Cl})\end{array}$ & $\begin{array}{c}\text { Painful TMD } \\
\text { muscle + joint } \\
n(\%)\end{array}$ & $\begin{array}{c}\mathrm{RR} \\
(95 \% \mathrm{Cl})\end{array}$ & $\begin{array}{l}\text { Total } \\
\text { n (\%) }\end{array}$ \\
\hline no & $\begin{array}{c}626 \\
(64.1)\end{array}$ & $\begin{array}{c}86 \\
(26.1)\end{array}$ & (ref.) & $\begin{array}{c}11 \\
(33.3)\end{array}$ & (ref.) & $\begin{array}{c}48 \\
(29.3)\end{array}$ & (ref.) & $\begin{array}{c}27 \\
(20.3)\end{array}$ & (ref.) & $\begin{array}{c}712 \\
(54.5)\end{array}$ \\
\hline yes & $\begin{array}{c}351 \\
(35.9)\end{array}$ & $\begin{array}{c}244 \\
(73.1)\end{array}$ & $\begin{array}{c}1.490 \\
(1.386-1.602) \\
{ }^{*} p<0.0001\end{array}$ & $\begin{array}{c}22 \\
(66.4)\end{array}$ & $\begin{array}{c}1.044 \\
(1.016-1.073) \\
{ }^{*} p=0.0007\end{array}$ & $\begin{array}{c}116 \\
(70.7)\end{array}$ & $\begin{array}{c}1.236 \\
(1.168-1.307) \\
{ }^{*} p<0.0001\end{array}$ & $\begin{array}{c}106 \\
(79.7)\end{array}$ & $\begin{array}{c}1.248 \\
(1.184-1.316) \\
{ }^{*} p<0.0001\end{array}$ & $\begin{array}{c}595 \\
(45.5)\end{array}$ \\
\hline Total & $\begin{array}{c}977 \\
(100.0)\end{array}$ & $\begin{array}{c}330 \\
(100.0)\end{array}$ & & $\begin{array}{c}33 \\
(100.0)\end{array}$ & & $\begin{array}{c}164 \\
(100.0)\end{array}$ & & $\begin{array}{c}133 \\
(100.0)\end{array}$ & & $\begin{array}{c}1307 \\
(100.0)\end{array}$ \\
\hline
\end{tabular}

ref. $=$ reference values. ${ }^{*}$ chi-square test

Table 4. Risk for chronic painful TMD ( $>3$ months) according to HAc

\begin{tabular}{|c|c|c|c|c|c|c|c|c|}
\hline \multicolumn{9}{|c|}{ Chronic painful TMD } \\
\hline $\mathrm{HAc}$ & $\begin{array}{c}\text { No TMD } \\
+ \text { non painful TMD } \\
\mathrm{n}(\%)\end{array}$ & $\begin{array}{c}\text { Painful TMD } \\
\text { overall } \\
\mathrm{n}(\%) \\
\end{array}$ & $\begin{array}{c}\mathrm{RR} \\
(95 \% \mathrm{Cl})\end{array}$ & $\begin{array}{c}\text { Acute TMD } \\
\mathrm{n}(\%)\end{array}$ & $\begin{array}{c}\text { RR } \\
(95 \% \mathrm{Cl})\end{array}$ & $\begin{array}{c}\text { Chronic TMD } \\
n(\%)\end{array}$ & $\begin{array}{c}\text { RR } \\
(95 \% \mathrm{Cl})\end{array}$ & $\begin{array}{l}\text { Total } \\
\mathrm{n}(\%)\end{array}$ \\
\hline no & $\begin{array}{c}626 \\
(64.1)\end{array}$ & $\begin{array}{c}86 \\
(26.1)\end{array}$ & (ref.) & $\begin{array}{c}30 \\
(36.1)\end{array}$ & (ref.) & $\begin{array}{c}56 \\
(22.7)\end{array}$ & (ref.) & $\begin{array}{c}712 \\
(54.5)\end{array}$ \\
\hline yes & $\begin{array}{c}351 \\
(35.9)\end{array}$ & $\begin{array}{c}244 \\
(73.9)\end{array}$ & $\begin{array}{c}1.490 \\
(1.386-1.602) \\
{ }^{*} p<0.0001\end{array}$ & $\begin{array}{c}53 \\
(63.9)\end{array}$ & $\begin{array}{c}1.098 \\
(1.1054-1.145) \\
{ }^{*} p<0.0001\end{array}$ & $\begin{array}{c}191 \\
(77.3)\end{array}$ & $\begin{array}{c}1.417 \\
(1.327-1.514) \\
{ }^{*} p<0.0001\end{array}$ & $\begin{array}{c}595 \\
(45.5)\end{array}$ \\
\hline Total & $\begin{array}{c}977 \\
(100.0)\end{array}$ & $\begin{array}{c}330 \\
(100.0)\end{array}$ & & $\begin{array}{c}83 \\
(100.0)\end{array}$ & & $\begin{array}{c}247 \\
(100.0)\end{array}$ & & $\begin{array}{c}1307 \\
(100.0)\end{array}$ \\
\hline
\end{tabular}

ref.= reference values. ${ }^{*}$ chi-square test

increased the risk in higher magnitude for chronic TMD pain $(1.41 ; 1.32-1.51)$ (Table 4$)$.

\section{DISCUSSION}

The first finding of the present study refers to the high prevalence rates of HAc and TMD. According to literature, headache prevalence are up to $51 \%$ in children/ adolescents, ${ }^{(5)}$ although mostly rates are of $2 \%$ to $5 \% .{ }^{(6)}$ Brazilian data about TMD and HAc in adolescents agree with international rates for headaches prevalence but not for TMD, which was surprisingly very high (about 30\%). However, a recent study with adolescents from Mexico demonstrated similar prevalence of TMD, with 33.2\%. ${ }^{(7)}$ The reasons which may underlie this higher prevalence should be further explored.

The second finding of this study refers to gender differences. After stratifying adolescents by gender, females demonstrated significant risks for the presence of HAc but not for TMD, as similar previously observed in adults. ${ }^{(3)}$ However, in the presence of both conditions, higher risks were observed for girls with menarche. Based on this find, we suggest that the hormonal related pain changes should be explored in the future. It would be important to verify how these changes affect the relationship between headache and TMD, since most of researches exploring gender differences in pain never consider these conditions together.

Although a strong association between headaches and TMD has been demonstrated, this relationship is still poorly explored in adolescents. Considering TMD and HA pain characteristics, it becomes also evident that the relationship between them is complex, from both pathophysiological and clinical perspectives. Basically, there are two hypotheses to justify the association. Clinically, TMD pain can characterize a "secondary headache", since the temporalis is a masticatory muscle located in the head. Considering pathophysiology, the comorbidity of these two chronic pain conditions seems to be related to central sensitization, ${ }^{(8)}$ especially in trigeminal subnucleus caudalis.

When interpreting the results, some points deserve attention. First, HAc are based on adolescents responses to one question, and consequently, no headache diagnosis could be attributed. Second, a cross sectional study allows the detection of association, but no causal 
relationship can be established. Strengths include the standardized methodology for epidemiology and for TMD classification.

It could be concluded that HAc increased the risk for TMD in adolescents, especially in girls with menarche. The risk was particular higher for painful and chronic TMD.

\section{REFERENCES}

1. LeResche L, Mancl LA, Drangsholt MT et al. Predictors of onset of facial pain and temporomandibular disorders in early adolescence. Pain. 2007;129(3):269-78.

2. Bertoli FMP, Antoniuk SA, Bruck I et al. Evaluation of the signs and symptoms of temporomandibular disorders in children with headaches. Arq. Neuro-Psiquiatr. 2007;65(2A):251-5.

3. Franco AL, Gonçalves DA, Castanharo SM, et al. Migraine is the most prevalent primary headache in individuals with temporo mandibular disorders. J Orofac Pain. 2010;24 (3):287-92.
4. Dworkin SF, LeResche L. 1992. Research diagnostic criteria for temporomandibular disorders: review, criteria, examinations and specifications, critique. J Craniomand Disorders. 1992; 6(4): $301-55$

5. Stovner LJ, Hagen K, Jensen R et al. The global burden of headache: a documentation of headache prevalence and disability worldwide. Cephalalgia. 2007;27(3):193-210.

6. Nilsson IM, List T, Drangsholt M. Incidence and temporal patterns of temporomandibular disorder pain among Swedish adolescents. J Orofac Pain. 2007;21 (2):127-32.

7. Moyaho-Bernal A, Lara-Muñoz M C, Espinosa-De Santilhana l et al. Prevalence of signs and symptoms of temporomandibular disorders in children in the state of Puebla, Mexico, evaluated with the Research Diagnostic Criteria for Temporomandibular Disorders (RDC/TMD). Acta Odontol Latinoam. 2010;23 (3): 228-33.

8. Woolf CJ. Central sensitization: implications for the diagnosis and treatment of pain. Pain. 2011;152(3 Suppl): S2-S15. 